\title{
Can Open Access Journals Help Uphold the Value of Research Integrity?
}

\section{Nhung T. Nguyen}

Dept. of Management, College of Business \& Economics, Towson University, Towson

Recently, the New York Times published an article about a fraud case in which a psychologist from the Netherlands was reported to have published his research using falsified data [2]. This article should serve as a wakeup call to many of us, organizational researchers and journal editors alike. Although research misconduct has remained largely anecdotal in our field of business, and outright fabrication of an entire experiment for publication in top tier journals such as the case of Professor Diederik Stapel at Tilburg University in the Netherlands [2] has not been heard of in organizational science; indirect evidence of such research misconduct has recently been reported among management researchers [1]. It is high time we addressed the issue of research integrity at the Journal of Business and Financial Affairs while the journal is still in its infancy. We certainly do not want to be in the position of highly respected journals such as the Science where Professor Stapel published an article based on a fraudulent dataset. In this commentary, I will discuss open access as a potential solution to mitigate misconduct in organizational research.

As journal editors, I think there are a few things we can do to proactively prevent fraudulent research from being published. First, we need to establish a written policy on research ethics guidelines, procedures concerning how we respond to charges of research misconduct. In the letter to the Editor, authors need to provide a statement of having read and agreed to our journal policy and procedures before having their manuscripts submitted for peer review. I hope that this will increase author's awareness and perception concerning research misconduct that will subsequently prevent author's intention to engage in such misconduct. There is evidence of this effect on student views concerning plagiarism based on a longitudinal study [4] and I hope the same pattern of results can be generalized to organizational researchers.

Second, we need re-define the meaning of value added research. I know that at many colleges and universities especially large universities, basic research is more valued than applied research. This creates a lot of pressure among faculty to abandon research that might be just a replication study in pursuit of the so-called "novel" research that will be more rewarded in terms of promotion and tenure as well as merit allocation. Needless to say, the pressure to pursue basic research has led to more data fabrication and/or falsification to publish one's research. In a recent survey on research misconduct among 384 faculty members at AACSB colleges in the U.S. it was reported that almost $73 \%$ of surveyed faculty having knowledge of their colleagues in the past year engaging in data falsification, fabrication, or plagiarism [1]. More astonishingly, tenured and tenure-track faculty were equally likely to commit research fraud contrary to our assumption that tenure-track faculty would be under more pressure to publish compared to tenured faculty [1]. Thus, if we make it our journal policy to publish not only statistically significant results but statistically non-significant ones, I think the pressure to fabricate data to make them significant will be reduced.

Third, if we encourage authors by publishing their replication studies, the pressure to conduct basic research to develop new theories and models will be lessened, and consequently, research misconduct less likely. Open access journals play an important role in this regard as open access allows researchers to share research findings and reach a much larger audience compared to traditional subscription based journals. Open access will enable research synthesis as meta-analysts will have an easier access to primary studies. Thus, open access improves the accuracy of research synthesis because it mitigates the file drawer problem defined as unpublished research because such research contains statistically non-significant findings.

However hard we as journal editors try to establish and enforce our policy of research integrity, it is still possible for authors to commit fraud as it has been and will be an honor system in which we expect ourselves as well as our colleagues to operate when it comes to conducting and publishing research. Our journal, like others, needs to rely on reviewers when it comes to evaluating research manuscripts. It would be an undue burden to ask our reviewers to play the role of policemen when it comes to detecting research misconduct. Despite my prior claim of reducing research misconduct by asking authors to sign a statement before submitting their work, we need to be aware that it will still be possible for authors to sneak in a manuscript based on a fraudulent dataset.

Thus, it is my opinion that unless we change our reward policy (e.g. promotion and tenure based on research rather than teaching; and basic research is more valued than applied research) we will perpetuate "the folly of rewarding A while hoping for B" as Steven Kerr poignantly stated some thirty some years ago [3] and that research misconduct will still be published undetected. However, it is also my belief that if our journal enforces some of the above recommendations, we will at least move forward with our effort to proactively maintain the credibility of our research discipline.

\section{References}

1. Bedeian A G, Taylor S G, Miller A N (2010) Management science on the credibility bubble: Cardinal sins and various misdemeanors. Academy Of Management Learning \& Education 9: 715-725.

2. Carey B (2011) Fraud Case Seen as a Red Flag for Psychology Research. The New York Times $\mathrm{p} 3$

3. Kerr S (1975) On the folly of rewarding A while hoping for B. Academy of management journal 18: 761-783.

4. Sims R L (2002) The Effectiveness of a Plagiarism Prevention Policy: A Longitudinal Study of Student Views. Teaching Business Ethics 6: 477-482.

*Corresponding author: Nhung T. Nguyen, Dept. of Management, College of Business \& Economics, Towson University, Towson, USA, Tel: 410704 2900; Fax: 410663 0207; E-mail: nnguyen@towson.edu

Received November 29, 2011; Accepted December 03, 2011; Published December 06, 2011

Citation: Nguyen NT (2012) Can Open Access Journals Help Uphold the Value of Research Integrity? J Bus \& Fin Aff 1:e102. doi:10.41722167-0234/.1000e102

Copyright: (c) 2012 Nguyen NT. This is an open-access article distributed under the terms of the Creative Commons Attribution License, which permits unrestricted use, distribution, and reproduction in any medium, provided the original author and source are credited. 